\title{
Maternal food choices in pregnancy influence epigenetic patterns in the offspring: results from the ROLO randomised control trial
}

\author{
A.A. Geraghty ${ }^{1}$, A. Sexton-Oates ${ }^{2}$, E.C. O’Brien ${ }^{1}$, G. Alberdi ${ }^{1}$, P. Fransquet ${ }^{2}$, R. Saffery ${ }^{2,3}$ and \\ F.M. McAuliffe \\ ${ }^{1}$ UCD Perinatal Research Centre, Obstetrics and Gynaecology, School of Medicine, University College Dublin National \\ Maternity Hospital, Dublin 2, Ireland \\ ${ }^{2}$ Cancer and Disease Epigenetics, Murdoch Childrens Research Institute, Melbourne, Victoria, Australia and \\ ${ }^{3}$ Department of Paediatrics, University of Melbourne, Victoria, Australia
}

A mixture of social, personal and environmental factors affect food choices however research shows that pregnancy is a powerful stimulus for positive changes ${ }^{(1)}$. The epigenetic profile of the developing fetus is sensitive to environmental influence. Mounting research suggests a role of variable DNA methylation in fetal programming of risk for conditions such as obesity and cardiovascular diseases $^{(2,3)}$. Maternal diet has been shown to influence individual DNA methylation patterns in offspring but research in humans is limited to observational studies with poorly defined exposures. The aim of this study was to investigate the impact of a dietary intervention to alter maternal food choices during pregnancy on neonatal epigenetic profiles.

Sixty neonates from the ROLO study (Randomised cOntrol trial of a LOw Gycaemic Index diet in pregnancy to prevent macrosomia $^{(3)}$ were selected. DNA methylation was investigated in $771,484 \mathrm{CpG}$ sites in cord blood. Principal component analysis was undertaken and epigenome-wide associations were examined using linear regression for exposure to the intervention. Associations with maternal and offspring factors were also explored.

Principal component and regression analyses identified widespread variation in the DNA methylation profile of newborns of mothers that changed to the low glycaemic index diet, accounting for $11 \%$ of total variation. No association was found with maternal early-pregnancy body mass index, infant sex, or birthweight. Pathway analysis identified influences of the dietary intervention on various aspects of offspring cellular function including pancreatic and immune functioning.

We identified preliminary evidence of widespread differential methylation in progeny of mothers following a low glycaemic index diet. This included differential methylation of several genes plausibly linked to pathways targeted by the intervention. With maternal food choices in pregnancy being driven by desire for a healthy pregnancy outcomes, these results could influence what women chose to eat during their pregnancy. Healthy dietary choices in pregnancy are vital to break the cycle of transmission of poor metabolic health from mother to offspring via epigenetic variation.

1. O'Brien OA, Lindsay KL, McCarthy M et al. (2017) Midwifery 47, 28-35.

2. Rhee KE, Phelan S, McCaffery J. (2012) Int J Pediatr 1-9.

3. Smith CJ, Ryckman KK. (2015) Diabetes 8, 295-302.

4. Walsh JM, McGowan CA, Mahony R et al. (2012) Br Med J 345, e5605-e5605. 